\title{
Re-emergence of enterovirus D68 in Europe after easing the COVID-19 lockdown, September 2021
}

Kimberley SM Benschop ${ }^{1}$, Jan Albert ${ }^{2,3}$, Andres Anton ${ }^{4}$, Cristina Andrés ${ }^{4}$, Maitane Aranzamendi5 , Brynja Armannsdóttir 6 , Jean-Luc Bailly7,8 , Fausto Baldanti ${ }^{9,10}$, Guðrún Erna Baldvinsdóttir ${ }^{6}$, Stuart Beard ${ }^{11}$, Natasa Berginc ${ }^{12}$, Sindy Böttcher ${ }^{13}$, Soile Blomqvist ${ }^{14}$, Laura Bubba ${ }^{15}$, Cristina Calvo ${ }^{16}$, Maria Cabrerizo ${ }^{17}$, Annalisa Cavallero $^{18}$, Cristina Celma ${ }^{11}$, Ferruccio Ceriotti ${ }^{19}$, Inês Costa ${ }^{20}$, Simon Cottrell ${ }^{21}$, Margarita del Cuerpo ${ }^{22}$, Jonathan Dean ${ }^{23}$, Jennifer L Dembinski ${ }^{24}$, Sabine Diedrich $^{13}$, Javier Diez-Domingo ${ }^{25}$, DagnyHaug Dorenberg ${ }^{24}$, Erwin Duizer ${ }^{1}$, Robert Dyrdak ${ }^{2,3}$, Diana Fanti ${ }^{26}$, Agnes Farkas ${ }^{27}$, Susan Feeney ${ }^{28}$, Jacky Flipse ${ }^{29}$ , Cillian De Gascun ${ }^{23}$, Cristina Galli ${ }^{15}$, Irina Georgieva ${ }^{30}$, Laura Gifford ${ }^{21}$, Raquel Guiomar ${ }^{20}$, Mario Hönemann ${ }^{31}$, Niina Ikonen ${ }^{14}$, Marion Jeannoël ${ }^{32}$, Laurence

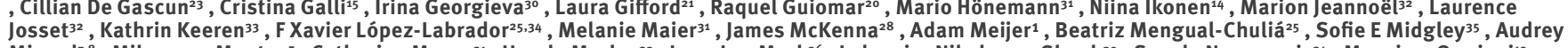
Mirand $^{7,8}$, Milagrosa Montes ${ }^{5}$, Catherine Moore ${ }^{21}$, Ursula Morley ${ }^{23}$, Jean-Luc Murk ${ }^{36}$, Lubomira Nikolaeva-Glomb ${ }^{30}$, Sanela Numanovic ${ }^{24}$, Massimo Oggioni ${ }^{19}{ }^{2}$, Paula Palminha ${ }^{20}$, Elena Pariani ${ }^{15}$, Laura Pellegrinelli ${ }^{15}$, Antonio Piralla9 , Corinna Pietsch ${ }^{31}$, Luis Piñeiro ${ }^{5}$, Núria Rabella ${ }^{22}$, Petra Rainetova ${ }^{37}$, Sara Colonia Uceda Renteria $^{19}$, María P Romero ${ }^{15}$, Marijke Reynders ${ }^{37}$, Lieuwe Roorda ${ }^{39}$, Carita Savolainen-Kopra ${ }^{14}$, Isabelle Schuffenecker ${ }^{32}$, Aysa Soynova ${ }^{30}$, Caroline MA Swanink ${ }^{29}$

, Tina Ursic $4^{40}$, Jaco J Verweij ${ }^{36}$, Jorgina Vila ${ }^{41}$, Tytti Vuorinen ${ }^{42}$, Peter Simmonds ${ }^{43}$, Thea K Fischer ${ }^{44,45, *}$, Heli Harvala ${ }^{46,47, *}$

1. Center for Infectious Disease Control, National Institute for Public Health and the Environment, Bilthoven, the Netherlands

2. Department of Clinical Microbiology, Karolinska University Hospital, Stockholm, Sweden

3. Department of Microbiology, Tumor and Cell Biology, Karolinska Institutet, Stockholm, Sweden

4. Respiratory Virus Unit, Microbiology Department, Vall d'Hebron Institut de Recerca (VHIR), Vall d'Hebron Barcelona Hospital Campus, Barcelona, Spain

5. Microbiology Department, Donostia University Hospital and Biodonostia Health Research Institute, San Sebastián, Spain

6. Landspitali-National University Hospital, Reykjavik, Iceland

7. CHU Clermont-Ferrand, National Reference Centre for enteroviruses and parechoviruses - Associated laboratory, Clermont-Ferrand, France

8. Université d’Auvergne, LMGE UMR CNRS 6023, Equipe EPIE - Epidémiologie et physiopathologie des infections à entérovirus, Faculté de Médecine, ClermontFerrand, France

9. Microbiology and Virology Department, Fondazione IRCCS Policlinico San Matteo, Italy

10. Department of Clinical Surgical Diagnostic and Pediatric Sciences, Università degli Studi di Pavia, Pavia, Italy

11. UK Health Security Agency, Colindale, United Kingdom

12. National laboratory of health, environment and food, Laboratory for public health virology, Ljubljana, Slovenia

13. National Reference Center for Poliomyelitis and Enteroviruses, Robert-Koch Institute, Berlin, Germany

14. National Institute for Health and Welfare, Helsinki, Finland

15. Department of Biomedical Sciences of Health, University of Milan, Milan, Italy

16. Hospital Universitario La Paz, Madrid, Spain

17. National Centre for Microbiology, Instituto de Salud Carlos III, Enterovirus and Viral Gastroenteritis Unit/Polio National Lab, Madrid, Spain

18. Laboratory of Microbiology, ASST Monza, San Gerardo Hospital, Monza (MB), Italy

19. Virology Unit, Division of Clinical Laboratory, Fondazione IRCCS Ca' Granda Ospedale Maggiore Policlinico, Milan, Italy

20. National Institute of Health (INSA), Lisbon, Portugal

21. Public Health Wales, Cardiff, United Kingdom

22. Microbiology Department Hospital Universitari de la Santa Creu i Sant Pau, Universitat Autònoma de Barcelona, Barcelona, Spain

23. National Virus Reference Laboratory, University College Dublin, Dublin, Ireland

24. Department of Virology, Norwegian Institute of Public Health, Oslo, Norway

25. Center for Public Health Research (FISABIO-Public Health), Generalitat Valenciana, Valencia, Spain

26. Chemical-clinical and Microbiological Analyses, ASST Grande Ospedale Metropolitano Niguarda, Milan, Italy

27. National Public Health Center, Budapest, Hungary

28. Regional Virus Laboratory, Belfast Health and Social Care Trust (BHSCT, Royal Victoria Hospital, Belfast, United Kingdom

29. Laboratory for Medical Microbiology and Immunology, Rijnstate, Velp, the Netherlands

30. National Reference Laboratory for Enteroviruses, National Center of Infectious and Parasitic Diseases, Sofia, Bulgaria

31. Institute of Medical Microbiology and Virology, University of Leipzig, Leipzig, Germany

32. National Reference Center for Enteroviruses and Parechoviruses, Institut des Agents Infectieux, Hospices Civils de Lyon, Lyon, France

33. Secretary of the commission for Polio Eradication in Germany, Robert-Koch Institute, Berlin, Germany

34. CIBERESP, Instituto de Salud Carlos III, Madrid, Spain

35. The Danish WHO National Reference Laboratory for Poliovirus, Statens Serum Institut, Copenhagen, Denmark

36. Elisabeth Tweesteden Hospital, Tilburg, the Netherlands

37. National Institute of Public Health, Prague, Czech Republic

38. Laboratory Medicine, Molecular Microbiology, AZ St.Jan Brugge-Oostende AV, Bruges, Belgium

39. Maasstad Hospital, Rotterdam, the Netherlands

40. Institute of Microbiology and Immunology, Faculty of Medicine, University of Ljubljana, Ljubljana, Slovenia

41. Pediatric Hospitalization Unit, Department of Pediatrics, Hospital Universitari Vall d'Hebron, Vall d'Hebron Barcelona Hospital Campus, Barcelona, Spain

42. Clinical Microbiology, Turku University Hospital and Institute of Biomedicine, University of Turku, Turku, Finland

43. University of Oxford, Oxford, United Kingdom

44. University of Sothern Denmark, Odense, Denmark

45. Nordsjaellands Hospital, Hillerod, Denmark

46. NHS Blood and Transplant, Microbiology Services, Colindale, United Kingdom

47. University College London (UCL), Department of infection and Immunity, London, United Kingdom

* These authors contributed equally and share last authorship.

\section{Correspondence: Kimberley S.M. Benschop (kim.benschop@rivm.nl)}

Citation style for this article:

Benschop Kimberley SM, Albert Jan, Anton Andres, Andrés Cristina, Aranzamendi Maitane, Armannsdóttir Brynja, Bailly Jean-Luc, Baldanti Fausto, Baldvinsdóttir Guðrún Erna, Beard Stuart, Berginc Natasa, Böttcher Sindy, Blomqvist Soile, Bubba Laura, Calvo Cristina, Cabrerizo Maria, Cavallero Annalisa, Celma Cristina, Ceriotti Ferruccio, Costa Inês, Cottrell Simon, del Cuerpo Margarita, Dean Jonathan, Dembinsk Jifer, Diedrich Sabine, Diez-Domingo Javier, Dorenberg Dagny Haug, Duizer Erwin, Dyrdak Robert, Fanti Diana, Farkas Agnes, Feeney Susan, Flipse Jacky, De Gascun Ciltian, Galti Cristina, Georgieva lrina, Gifford Laura, Guiomar Raquel, Hönemann Mario, Ikonen Nina, Jeannoelt Marion, Josset Laurence, Keeren Kathrin, López-Labrado FXavier, Maier Melanie, McKenna James, Meijer Adam, Mengual-Chuliá Beatriz, Midgley Sofie E, Mirand Audrey, Montes Milagrosa, Moore Catherine, Morley Ursula, Murk Jean-Luc, Nikolaeva-Glomb Lubomira, Numanovic Sanela, Oggioni Massimo, Palminha Paula, Pariani Elena, Pellegrinelli Laura, Piralla Antonio, Pietsch Corinna, Piñeiro Luis, Rabella Núria, Raineto Verweii Jaco I, Vila Jorgina, Vuorinen Tytti, Simmonds Peter, Fischer Thea K, Harvala Heli. Re-emergence of enterovirus D68 in Europe after easing the COVID-19 lockdown, September 2021. Euro Surveill. 2021;26(45):pii=2100998. https://doi.org/10.2807/1560-7917.ES.2021.26.45.2100998

We report a rapid increase in enterovirus D68 (EVD68) infections, with 139 cases reported from eight European countries between 31 July and 14 October 2021. This upsurge is in line with the seasonality of EV-D68 and was presumably stimulated by the widespread reopening after COVID-19 lockdown. Most cases were identified in September, but more are to be expected in the coming months. Reinforcement of clinical awareness, diagnostic capacities and surveillance of EV-D68 is urgently needed in Europe. 
TABLE 1

Details for 36 institutions reporting to this study and number of EV-D68 cases identified via their enterovirus and EV-D68 surveillance systems, 18 European countries, 1 January-14 October 2021 ( $\mathrm{n}=139$ cases)

\begin{tabular}{|c|c|c|c|c|c|c|c|}
\hline Country & Code & $\begin{array}{l}\text { Type of } \\
\text { laboratory }\end{array}$ & $\begin{array}{l}\text { Enterovirus } \\
\text { surveillance }\end{array}$ & $\begin{array}{l}\text { Samples subjected to EV-D68 } \\
\text { testing }\end{array}$ & \begin{tabular}{|c|} 
Use of \\
EV-D68- \\
specific PCR
\end{tabular} & $\begin{array}{l}\text { Molecular EV } \\
\text { typing }\end{array}$ & $\begin{array}{l}\text { Number } \\
\text { of EV-D68 } \\
\text { cases }\end{array}$ \\
\hline Belgium & BE-01 & Hospital & NA & All clinical respiratory samples & Yes & No & 14 \\
\hline Bulgaria & BG-01 & Public Health & AFP and EVCS & All EV-positive samples & No & No & o \\
\hline Czechia & CZ-01 & Public Health & EVCS & Not currently in place & No & No & o \\
\hline \multirow{2}{*}{ Germany } & DE-01 & Public Health & EVCS & Limited respiratory samples $^{\mathrm{a}}$ & No & In-house & 0 \\
\hline & DE-02 & Academic Hospital & ILI/ARI & EV-positive samples & No & In-house & 0 \\
\hline Denmark & DK-01 & Public Health & ILI/ARI and EVCS & All EV-positive samples & No & In-house & 0 \\
\hline \multirow{6}{*}{ Spain } & ES-01 & Academic Hospital & EVCS & All EV-positive samples & No & In-house & 9 \\
\hline & ES-02 & Academic Hospital & EVCS & All EV-positive samples & No & In-house & 1 \\
\hline & ES-03 & Academic Hospital & NA & All EV-positive samples & No & ES-05 & 0 \\
\hline & ES-04 & Academic Hospital & NA & NK & NK & NK & 0 \\
\hline & ES-05 & Public Health & AFP and EVCS & All EV-positive samples & No & In-house & 0 \\
\hline & ES-06 & Public Health & ILI/ARI & EV-positive repiratory samples & No & In-house & o \\
\hline \multirow{2}{*}{ Finland } & $\mathrm{Fl}-01$ & Academic Hospital & NA & EV-positive samples & No & In-house & 0 \\
\hline & $\mathrm{Fl}-02$ & Public Health & ILI/ARI & EV-positive repiratory samples & Yes & In-house & o \\
\hline \multirow[t]{2}{*}{ France } & FR-01 & Academic Hospital & EVCS & $\begin{array}{l}\text { All EV-positive samples and } \\
\text { all HRV-EV positive respiratory } \\
\text { samples }\end{array}$ & No & In-house & 14 \\
\hline & FR-02 & Public Health & EVCS & All EV-positive samples & Yes & In-house & 6 \\
\hline Hungary & HU-01 & Public Health & AFP and EVCS & All EV-positive samples & No & In-house & o \\
\hline Ireland & $\mathrm{IE}-01$ & Public Health & EVCS & $\begin{array}{l}\text { Proportion of EV-positive } \\
\text { respiratory samples }\end{array}$ & Yes & In-house & 1 \\
\hline Iceland & IS-01 & Public Health & EVCS & All EV-positive samples & Yes & In-house & 0 \\
\hline \multirow{5}{*}{ Italy } & IT-01 & Public Health & ILI/ARI and AFP & All EV-positive samples & Yes & In-house & 1 \\
\hline & IT-02 & Academic Hospital & ILI/ARI & All clinical respiratory samples & Yes & In-house & 0 \\
\hline & IT-03 & Academic Hospital & $\mathrm{NA}$ & All clinical respiratory samples & No & IT-01/IT-02 & 0 \\
\hline & IT-04 & Hospital & NA & All clinical respiratory samples & No & IT-01/IT-02 & 0 \\
\hline & IT-05 & Hospital & NA & All clinical respiratory samples & No & IT-01/IT-02 & o \\
\hline \multirow{5}{*}{ The Netherlands } & NL-01 & Hospital & NA & All clinical respiratory samples & No & In-house & 1 \\
\hline & NL-02-A & \multirow{2}{*}{ Public Health } & EVCS & All EV-positive samples & No & In-house & 1 \\
\hline & $\mathrm{NL}-02-\mathrm{B}$ & & ILI/ARI & All clinical respiratory samples & Yes & In-house & 0 \\
\hline & NL-03 & Hospital & NA & All clinical respiratory samples & No & In-house & 0 \\
\hline & NL-04 & Hospital & NA & All clinical respiratory samples & No & In-house & o \\
\hline Norway & NO-01 & Public Health & AFP and EVCS & All EV-positive samples & Yes & In-house & 0 \\
\hline Portugal & PT-01 & Public Health & NA & All clinical respiratory samples & No & No & o \\
\hline Sweden & SE-01 & Academic Hospital & NA & $\begin{array}{l}\text { All clinical respiratory and } \\
\text { EV-positive samples }\end{array}$ & Yes & In-house ${ }^{b}$ & 2 \\
\hline \multirow{2}{*}{ Slovenia } & SI-01 & Public Health & ILI/ARI and AFP & All EV-positive samples & No & In-house & o \\
\hline & $\mathrm{SI}-02$ & Academic Hospital & NA & Mostly respiratory samples & Yes & In-house & o \\
\hline England, UK & UK-01 & Public Health & EVCS & All EV-positive samples & Yes & In-house & 7 \\
\hline Wales, UK & UK-02 & Public Health & ILI/ARI and EVCS & All EV-positive samples & Yes & In-house & 82 \\
\hline Belfast, UK & UK-03 & Hospital & NA & Any samples if clinically indicated & Yes & In-house & 0 \\
\hline
\end{tabular}

AFM: acute flaccid myelitis surveillance; AFP: acute flaccid paralysis surveillance; EV: enterovirus; EVCS: EV clinical surveillance (i.e. EV-positive clinical specimens are subjected to typing); HRV: human rhinovirus; ILI/ARI: sentinel influenza surveillance focusing on influenza like illness and acute respiratory infection; NA: not applicable; NK: not known; UK: United Kingdom.

a Only if respiratory sample has been taken from a case with acute flaccid paralysis.

${ }^{\mathrm{b}}$ EV-positive faecal and central nervous system samples referred for typing at public health agency. 


\section{TABLE 2}

Laboratory details for enterovirus detection and typing, 18 European countries, 1 January-14 October 2021 ( $\mathrm{n}=36$ laboratories)

\begin{tabular}{|c|c|c|c|}
\hline Country & Code & EV and screening methods & EV sequencing methods \\
\hline Belgium & BE-01 & $\begin{array}{c}\text { Faeces: EV PCR on GI-TAC assay; respiratory samples: EV PCR and EV-D68 PCR } \\
\text { on respiratory TAC assay; others: in-house EV PCR; CSF: FilmArray panel }\end{array}$ & NA \\
\hline Bulgaria & BG-01 & All samples: cell culture (A), and EV PCR (B) & NA \\
\hline Czechia & CZ-01 & Faeces: cell culture and EV PCR & NA \\
\hline \multirow{2}{*}{ Germany } & DE-01 & Only faeces and CSF tested, respiratory (only AFP cases): EV PCR & Complete or partial $\mathrm{VP}_{1}$ region \\
\hline & DE-02 & All samples: EV-PCR and EV/HRV PCR & Partial VP1 \\
\hline Denmark & DK-01 & All samples: EV PCR and HRV PCR & $\begin{array}{c}\text { Partial VP1 and VP4-VP2 for EV, VP2 } \\
\text { for RV }\end{array}$ \\
\hline \multirow{6}{*}{ Spain } & ES-01 & $\begin{array}{c}\text { All samples: HRV16 Allplex Respiratory Panel (Seegene) or RealCycle EV/hPeV } \\
\text { detection (Progenie) }\end{array}$ & Partial VP1 \\
\hline & ES-02 & Respiratory samples: Allplex Respiratory Panel; other samples: EV PCR & Partial VP1 \\
\hline & ES-03 & $\begin{array}{c}\text { All respiratory samples negative for other respiratory viruses, CSF and faeces } \\
\text { from neurological or cutaneous illnesses }\end{array}$ & Partial VP1 \\
\hline & ES-04 & No data & Partial VP1 \\
\hline & ES-05 & All samples: EV PCR & Partial VP1 \\
\hline & ES-06 & Respiratory samples: respiratory RT-PCR panel (EV/HRV in one channel) & EV-D68 typing PCR \\
\hline \multirow{2}{*}{ Finland } & $\mathrm{Fl}-01$ & Respiratory samples: EV/HRV PCR], All samples: EV-PCR & Partial VP1 \\
\hline & $\mathrm{Fl}-02$ & Respiratory samples: EV/HRV PCR and EV-D68 PCR & Complete $\mathrm{VP}_{1}$ and $\mathrm{VP}_{4}-\mathrm{VP}_{2}$ \\
\hline \multirow{3}{*}{ France } & FR-01 & Respiratory samples: EV/HRV PCR & $\begin{array}{c}\text { Complete or partial } \mathrm{VP}_{1} \text { and } \\
\text { VP4-VP2 }\end{array}$ \\
\hline & FR-02-A & All samples: EV PCR and EV/HRV PCR & \multirow{2}{*}{$\begin{array}{l}\text { Complete or partial } \mathrm{VP}_{1} \text { and } \\
\text { VP4-VP2; complete or partial } \mathrm{VP}_{1} \\
\text { and } \mathrm{VP}_{4}-\mathrm{VP}_{2}\end{array}$} \\
\hline & FR-02-B & $\begin{array}{l}\text { Respiratory samples ( } \$ 5 \text { years) or samples from severe cases (respiratory } \\
\text { AFM): EV-D68 PCR }\end{array}$ & \\
\hline Hungary & $\mathrm{HU}-01$ & All samples: EV PCR & 5'-NTR, partial VP1 \\
\hline Ireland & $\mathrm{IE}-01$ & $\begin{array}{l}\text { Respiratory samples: Luminex NxTAG Respiratory Panel (EV/HRV); respiratory } \\
\text { samples with clinical indication: EV-D68 PCR; all other samples: EV PCR }\end{array}$ & Partial VP1 \\
\hline Iceland & IS-01 & All samples: EV PCR and EV-D68 PCR & Partial VP1 \\
\hline \multirow{5}{*}{ Italy } & IT-01 & Respiratory samples: EV PCR and EV-D68 PCR & Partial VP1 and $\mathrm{VP}_{4}-\mathrm{VP}_{2}$ \\
\hline & IT-02 & Respiratory samples: EV/HRV PCR and EV-D68 PCR & $\begin{array}{c}\text { EV-D68 typing; } \mathrm{HRV} / \mathrm{EV} \text { on } \mathrm{VP} 4 / \mathrm{VP} 2 \\
\text { typing } \\
\text { VP1 typing }\end{array}$ \\
\hline & IT-03 & Respiratory samples: EV PCR: Allplex Respiratory Panel & NA \\
\hline & IT-04 & Respiratory samples: EV PCR: Allplex Respiratory Panel & NA \\
\hline & IT-05 & Respiratory samples: EV PCR: Allplex Respiratory Panel & NA \\
\hline \multirow{5}{*}{$\begin{array}{l}\text { The } \\
\text { Netherlands }\end{array}$} & NL-01 & All samples: EV PCR & Partial VP1 \\
\hline & NL-02-A & All samples: EV PCR & Partial VP1 \\
\hline & $\mathrm{NL}-02-\mathrm{B}$ & Respiratory samples: EV PCR and HRV PCR and EV-D68 PCR & 5'-NTR, EV-D68 VP1, partial VP1 \\
\hline & NL-03 & All samples: EV PCR & Partial VP1 \\
\hline & NL-04 & All samples: EV PCR & Partial VP1 \\
\hline Norway & NO-01 & All samples: EV PCR and EV-D68 PCR & Partial VP1 \\
\hline Portugal & PT-01 & Respiratory samples: EV PCR: Allplex Respiratory Panel & NA \\
\hline \multirow{2}{*}{ Sweden } & SE-01-A & Non-respiratory samples: EV PCR & Partial VP1 and VP4-VP2 \\
\hline & SE-01-B & Respiratory samples: Allplex Respiratory Panel and EV-D68 PCR & Partial VP1 and VP4-VP2 \\
\hline \multirow{2}{*}{ Slovenia } & SI-01 & Respiratory samples: EV PCR & Partial VP1 \\
\hline & $\mathrm{SI}-02$ & All samples: EV-D68 PCR & Partial VP1 \\
\hline England, UK & UK-01 & All samples: EV PCR and EV-D68 PCR & Partial VP1 \\
\hline Wales, UK & UK-02 & All respiratory samples/CNS/faeces: EV PCR and EV-D68 PCR & Partial VP1 \\
\hline Belfast, UK & UK-03 & $\begin{array}{c}\text { Respiratory samples with clinical indication; skin swab with clinical } \\
\text { indication; all CSF samples; blood with clinical symptoms; EV PCR and } \\
\text { EV-D68 PCR }\end{array}$ & $\begin{array}{l}\text { Partial VP1 Colindale reference } \\
\text { laboratory sequencing }\end{array}$ \\
\hline
\end{tabular}

AFM: acute flaccid myelitis; CSF: cerebrospinal fluid; EV: enterovirus; GI-TAC: gastrointestinal Taqman array card; hPeV: human parechovirus;

HRV: human rhinovirus; NA: not applicable; NTR: non-translated region; UK: United Kingdom; VP: viral protein.

References for the methods presented here are listed in the Supplement. 
FIGURE 1

EV-D68 detection in Europe, 1 July-14 October 2021 $(\mathrm{n}=139)$

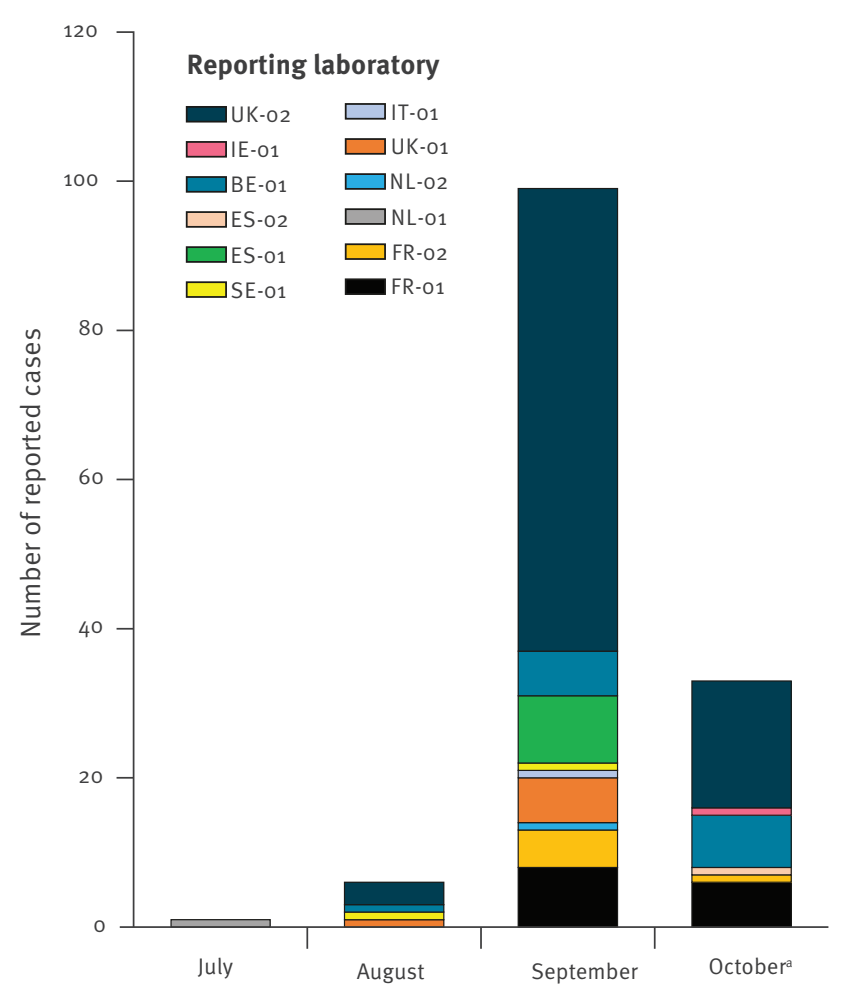

Sample collection on month

BE: Belgium; ES: Spain; EV: enterovirus; FR: France; IE: Ireland; IT: Italy; NL: the Netherlands; SE: Sweden; UK: United Kingdom.

a Data incomplete based on samples collected and reported as EVD68 by 14 October 2021.

Coordination of case reporting by the European NonPolio Enterovirus Network (ENPEN) found evidence for increasing numbers of enterovirus D68 (EV-D68) infections in Europe in September 2021. This prompted the consortium to send an alert email to member laboratories, requesting an urgent EV-D68 investigation. Here, we report the virological and clinical characteristics of 139 EV-D68 cases identified in eight European countries between 31 July and 14 October 2021.

\section{Enterovirus D68 detection and typing}

A total of 36 institutions including 19 public health and 17 hospital laboratories from 18 European countries responded to the ENPEN alert (Table 1).

We requested data on EV-D68 cases and detection methods. The 19 public health laboratories from 13 countries indicated that they would identify EV-D68 infections either via their EV surveillance $(n=14)$, surveillance focusing on influenza-like illness (ILI) and/ or acute respiratory infection (ARI; $n=8)$, or via surveillance for acute flaccid paralysis (AFP) $(n=6)$. Two laboratories (in the Czech Republic and Germany) had not included respiratory samples in their EV surveillance. Data on screening and typing were supplied by 33 laboratories. All except one of the 14 hospital laboratory tested respiratory samples for EV-D68. The use of EV-D68-specific PCR was reported by 13 of 33 laboratories, and genetic characterisation by sequencing was applied in most laboratories (28/33, Table 2).

\section{Distribution of enterovirus D68 cases in Europe}

A total of 139 EV-D68 cases were identified between 31 July and 14 October 2021 by 12 laboratories in eight countries (Table 1). Most EV-D68-positive samples were collected in September (99/139, 71\%, Figure). Screening and typing of samples collected in October is ongoing.

\section{Number of EV-D68 infections is increasing in Europe}

Denominator data were available from 24 laboratories reporting 66 of 139 EV-D68 cases. Since the beginning of 2021, these laboratories identified 1,964 EV-positive samples by screening 56,401 samples by EV PCR (some also detecting and hence including rhinoviruses in these reports). While the number of samples tested monthly remained unchanged (on average 6,113 samples screened monthly between January and July, 5,170 in August and 6,353 in September), an increase in the positivity rate was noted (overall $2.5 \%$ in January through July, $4.5 \%$ in August and $8.2 \%$ in September). A total of 967 samples were typed as EV and 36 as EV-D68. The proportion of samples identified as EV-D68 increased from $0.2 \%$ in January through July (1/409) and $0.9 \%$ in August (2/208) to $14 \%$ in September (33/236; $p<0.0001$ by chi-squared test).

An additional 30 EV-D68-positive samples were identified by screening of 8,243 respiratory samples by EV-D68-specific PCR in 13 laboratories. Of these, none was identified before August despite screening of 5,088 samples since January (monthly average: 727 samples). Three EV-D68 positive samples were identified in August $(3 / 739 ; 0.4 \%)$ and 27 in September and October $(27 / 1,289 ; 2 \%)$ demonstrating a recent but significant increase in the EV-D68 positivity rate ( $p<0.0001$ by chi-squared test).

\section{Clinical characteristics of enterovirus D68 cases}

Demographic information was collected for all reported EV-D68 cases (Table 3). Most were males (88/139; $63 \%$ ) and younger than 5 years (120/139; 86\%), with a median age of 3 years (range: newborns to 72 years). Clinical symptoms were reported for 120 cases, with most exhibiting respiratory symptoms $(n=116 ; 97 \%)$. Although five cases had neurological symptoms, none was diagnosed with AFP or acute flaccid myelitis (AFM). Thirty of 49 cases with data on hospitalisation were hospitalised. Pre-existing conditions were reported for 20 of 45 cases with available information, 


\section{TABLE 3}

Demographic and clinical characteristics of individuals with a laboratory-confirmed EV-D68 infection, eight European countries, 1 September-14 October 2021 $(\mathrm{n}=139)$

\begin{tabular}{|c|c|c|}
\hline & $\begin{array}{l}\text { Number } \\
\text { of cases }\end{array}$ & $\begin{array}{c}\text { Proportion } \\
\text { of cases }\end{array}$ \\
\hline \multicolumn{3}{|l|}{ Age group } \\
\hline $0-3$ months & 7 & $5 \%$ \\
\hline 4-12 months & 15 & $11 \%$ \\
\hline $13-24$ months & 22 & $16 \%$ \\
\hline $2-5$ years & 76 & $55 \%$ \\
\hline $6-15$ years & 9 & $6 \%$ \\
\hline $16-25$ years & 2 & $1 \%$ \\
\hline $26-45$ years & 2 & $1 \%$ \\
\hline$>45$ years & 6 & $4 \%$ \\
\hline \multicolumn{3}{|l|}{ Sex } \\
\hline Female & 51 & $37 \%$ \\
\hline Male & 88 & $63 \%$ \\
\hline \multicolumn{3}{|l|}{ Symptoms (data reported for) } \\
\hline Any symptom reported $(n=121)$ & 120 & $99 \%$ \\
\hline Fever $(n=111)$ & 49 & $44 \%$ \\
\hline Enteric symptoms $(\mathrm{n}=120)$ & 4 & $3 \%$ \\
\hline Respiratory symptoms $(\mathrm{n}=120)$ & 116 & $97 \%$ \\
\hline Neurological symptoms ${ }^{\mathrm{a}}(\mathrm{n}=111)$ & 5 & $5 \%$ \\
\hline \multicolumn{3}{|c|}{ Clinical information (data reported for) } \\
\hline Hospitalised $(n=49)$ & 30 & \\
\hline Pre-existing condition ${ }^{b}(n=45)$ & 20 & \\
\hline \multicolumn{3}{|l|}{ Co-infections (data reported for) } \\
\hline Any co-infection reported $(n=43)$ & 16 & \\
\hline Adenovirus & 4 & \\
\hline Rhinovirus & 6 & \\
\hline Human metapneumovirus & 1 & \\
\hline Adenovirus and rhinovirus & 1 & \\
\hline Adenovirus and bocavirus & 1 & \\
\hline Rhinovirus and SARS-CoV-2 & 1 & \\
\hline $\mathrm{PIV}_{4}$ and $\mathrm{CMV}$ & 1 & \\
\hline Rhinovirus, bocavirus and $\mathrm{PIV}_{3}$ & 1 & \\
\hline
\end{tabular}

CMV: cytomegalovirus; EV: enterovirus; PIV: parainfluenza virus; SARS-CoV-2: severe acute respiratory syndrome coronavirus 2.

a Reported neurological symptoms included headache, dizziness and agitation.

${ }^{b}$ Reported pre-existing conditions were asthma, chronic obstructive pulmonary disease, sleep apnoea and immunosuppression.

predominantly in older age groups (2/14<2 years, $18 / 31>2$ years). Viral co-infection was reported for 16 cases; the most common co-detection was rhinovirus $(n=9)$. To date, genotyping of 20 EV-D68 positive samples has shown all strains to be genotype B3 (data not shown).

\section{Discussion}

Enterovirus D68 (EV-D68) infections have been linked to AFP/AFM since a large outbreak associated with respiratory and neurological symptoms in children was described in North America in 2014 [1,2]. Although regular EV-D68 upsurges have been reported in Europe since 2010 [2-7], they largely ceased during the coronavirus disease (COVID-19) pandemic. Here we report EV-D68 circulation across Europe for the first time following the COVID-19 pandemic, with case numbers already exceeding what was reported during the most recent EV-D68 upsurge in 2019 [7].

Although EV-D68 circulation in Europe has largely followed a biennial epidemic pattern confined to the autumn season of even-numbered years, the autumn of 2019 showed an unexpected upsurge of EV-D68 infections leading to 93 reported cases, two with AFM, in five European countries [7]. EV-D68 has largely been detected through ILI/ARI sentinel surveillance because of its respiratory signature and in EV surveillance systems which have included respiratory samples since the occurrence of the first large outbreak of EV-D68 in Europe and North America where these samples were recommended $[8,9]$. Several institutes have additionally included EV-D68-specific PCR for their respiratory surveillance.

The timing of this increase in the number of EV-D68 infections is consistent with the known seasonality of EV, with numbers usually peaking in September and October [10]. However, this marked upsurge is likely to have been further precipitated by the widespread relaxation of COVID-19 mitigation measures such as travel restrictions, school closures, use of face masks and physical distancing. The findings are indeed consistent with the widespread resurgence of other community-transmitted respiratory infections, whose circulation in most of Europe had until recently largely ceased $[11,12]$. The interruption in the transmission of respiratory and enteric viruses, including EV-D68, has probably created large cohorts of susceptible young children without prior exposure or immunity to any such virus, potentially creating the conditions for largescale outbreaks of severe respiratory disease in this age group this winter.

As circulation of EV diminished during the lockdown, many of the surveillance systems not related to SARSCoV-2 were temporarily discontinued or received fewer specimens because testing facilities prioritised SARSCoV-2. Although most study participants have now re-started active investigations via established surveillance systems, it is also important to consider the minimum number of samples needed for effective surveillance. Nonetheless, laboratories in 16 countries use screening or surveillance systems that enable detection of EV-D68 infection through typing of EV-positive samples. At the time of our previous survey on the EV laboratory and surveillance capacity in Europe in 2016, only 11 countries had introduced or modified their existing surveillance systems to enable EV-D68 detection [8]. This clearly demonstrates increased capacity for detection and awareness of EV-D68 across Europe, probably a sign of the strength of the continued collaboration established through ENPEN $[9,13-16]$. Notably, 
the zero-reporting noted by two countries in this study was due to the exclusion of respiratory samples from their EV surveillance, an important reminder that a respiratory sample is needed for the detection of EV-D68 (even in cases of AFM) as the virus is only rarely detected in faecal or cerebrospinal fluid samples [9].

Our data clearly demonstrate that EV-D68 is now circulating in Europe, mostly affecting children or those with underlying conditions. Most paediatric cases presented with respiratory symptoms. Although no AFM cases were reported, we should be alert to the possibility of EV-D68-associated AFM cases occurring in the coming months following the rise in EV-D68 cases most evident in Wales, Belgium, France and Spain. The same trends were noted during the North American outbreak in 2014 where the majority of the initial cases were associated with respiratory diseases and neurological cases were only observed with a delay of a few weeks [1].

We recommend sequence analysis of EV-D68 to determine the relatedness of viruses circulating in Europe, and their potential link to a novel B3 subclade reported in 2019 [7]. The existing surveillance systems as well as laboratory and clinical networks relating to EV-D68 should be activated as this infection can have severe consequences [17-20].

\section{Conclusion}

Re-emergence of EV-D68, and its known association with several neurological infections, is a reminder that the surveillance for EV infections is important. This study shows that EV-D68 cases can be identified through a combination of ILI/ARI sentinel surveillance and EV surveillance expanded to include respiratory samples. It calls for continued careful monitoring and vigilant testing of respiratory samples.

\section{Acknowledgements}

The authors wish to thank the European Society for Clinical Virology for hosting the European Non-Polio Enterovirus Network (ENPEN). The authors would also like to thank everybody who contributed to the collection, testing and reporting of clinical and virological data presented in this study.

\section{Conflict of interest}

None declared.

\section{Authors' contributions}

$\mathrm{KB}$, TF and $\mathrm{HH}$ initiated the study. $\mathrm{KB}$ and $\mathrm{HH}$ wrote the first draft, prepared the tables and figures, as well as the final manuscript. PS and TF helped with drafting the manuscript. JA, AA, CA, MA, BA, JB, FB, GB, SuB, NB, SiB, SoB, LB, CC, $M C, A C, C C, F C, I C, S C, M C, J D$, JLD, SD, JDD, DD, ED, RD, DF, $A G, S F, J F, C D G, C G, I G, L G, R G, M H, N I, M J, L J, K K, X L, M e M$, JM, AdM, BM, SM, AuM, MiM, CM, UM, JM, LN, SN, MO, PP, $E P, L P, A P, C P, L P, N R, P R, S C, M R, M R, L R, C S, I S, A S, C S, T U$, JJV, JV and TV were responsible for testing and data collection. All other authors provided and checked data, critically reviewed and edited manuscript. All authors have accepted the final version of manuscript.

\section{References}

1. Aliabadi N, Messacar K, Pastula DM, Robinson CC, Leshem E, Sejvar J], et al. Enterovirus D68 Infection in Children with Acute Flaccid Myelitis, Colorado, USA, 2014. Emerg Infect Dis. 2016;22(8):1387-94. https://doi.org/10.3201/eid2208.151949 PMID: 27434186

2. Midgley CM, Watson JT, Nix WA, Curns AT, Rogers SL, Brown $B A$, et al. Severe respiratory illness associated with a nationwide outbreak of enterovirus D68 in the USA (2014): a descriptive epidemiological investigation. Lancet Respir Med. 2015;3(11):879-87. https://doi.org/10.1016/S22132600(15)00335-5 PMID: 26482320

3. Kramer R, Sabatier M, Wirth T, Pichon M, Lina B, Schuffenecker I, et al. Molecular diversity and biennial circulation of enterovirus D68: a systematic screening study in Lyon, France, 2010 to 2016. Euro Surveill. 2018;23(37):1700711. https:// doi.org/10.2807/1560-7917.ES.2018.23.37.1700711 PMID: 30229724

4. Holm-Hansen CC, Midgley SE, Fischer TK. Global emergence of enterovirus D68: a systematic review. Lancet Infect Dis. 2016;16(5):e64-75. https://doi.org/10.1016/S14733099(15)00543-5 PMID: 26929196

5. Cottrell S, Moore C, Perry M, Hilvers E, Williams C, Shankar AG. Prospective enterovirus D68 (EV-D68) surveillance from September 2015 to November 2018 indicates a current wave of activity in Wales. Euro Surveill. 2018;23(46):1800578. https:// doi.org/10.2807/1560-7917.ES.2018.23.46.1800578 PMID: 30458915

6. Meijer A, van der Sanden S, Snijders BE, Jaramillo-Gutierrez G, Bont L, van der Ent CK, et al. Emergence and epidemic occurrence of enterovirus 68 respiratory infections in The Netherlands in 2010. Virology. 2012;423(1):49-57. https://doi. org/10.1016/j.virol.2011.11.021 PMID: 22177700

7. Midgley SE, Benschop K, Dyrdak R, Mirand A, Bailly JL, Bierbaum S, et al. Co-circulation of multiple enterovirus D68 subclades, including a novel B3 cluster, across Europe in a season of expected low prevalence, 2019/20. Euro Surveill. 2020;25(2):1900749. https://doi.org/10.2807/1560-7917. ES.2020.25.2.1900749 PMID: 31964463

8. Harvala H, Jasir A, Penttinen P, Pastore Celentano L, Greco D, Broberg E. Surveillance and laboratory detection for non-polio enteroviruses in the European Union/European Economic Area, 2016. Euro Surveill. 2017;22(45):16-00807. https:// doi.org/10.2807/1560-7917.ES.2017.22.45.16-00807 PMID: 29162204

9. Harvala H, Broberg E, Benschop K, Berginc N, Ladhani S, Susi $P$, et al. Recommendations for enterovirus diagnostics and characterisation within and beyond Europe. J Clin Virol. 2018;101:11-7. https://doi.org/10.1016/j.jcv.2018.01.008 PMID: 29414181

10. Bubba L, Broberg E, Jasir A, Simmonds P, Participants ES, Harvala $H$. Circulation of non-polioviruses in 24 EU/EAA countries between 2015 and 2017 - is it time to consider systematic surveillance? Lancet Infect Dis. 2019. Forthcoming.

11. van Summeren J, Meijer A, Aspelund G, Casalegno JS, Erna G, Hoang U, et al. Low levels of respiratory syncytial virus activity in Europe during the 2020/21 season: what can we expect in the coming summer and autumn/winter? Euro Surveill. 2021;26(29):2100639. https://doi.org/10.2807/1560-7917. ES.2021.26.29.2100639 PMID: 34296672

12. von Hammerstein AL, Aebi C, Barbey F, Berger C, Buettcher M Casaulta C, et al. Interseasonal RSV infections in Switzerland - rapid establishment of a clinician-led national reporting system (RSV EpiCH). Swiss Med Wkly. 2021;151:w30057. PMID: 34499459

13. Poelman R, Schuffenecker I, Van Leer-Buter C, Josset L, Niesters HG, Lina BEuropean surveillance for enterovirus D68 during the emerging North-American outbreak in 2014. J Clin Virol. 2015;71:1-9. https://doi.org/10.1016/j.jcv.2015.07.296 PMID: 26364237

14. Benschop KSM, Broberg EK, Hodcroft E, Schmitz D, Albert J, Baicus A, et al. Molecular epidemiology and evolutionary trajectory of emerging echovirus 30, Europe. Emerg Infect Dis. 2021;27(6):1616-26. https://doi.org/10.3201/eid2706.203096 PMID: 34013874

15. Harvala H, Benschop KSM, Berginc N, Midgley S, Wolthers $\mathrm{K}$, Simmonds $\mathrm{P}$, et al. European non-polio enterovirus network: introduction of hospital-based surveillance network to understand the true disease burden of nonpolio enterovirus and parechovirus infections in Europe. 
Microorganisms. 2021;9(9):1827. https://doi.org/10.339o/ microorganisms9091827 PMID: 34576722

16. Fischer TK, Simmonds $P$, Harvala $H$. The importance of enterovirus surveillance in a post-polio world. Lancet Infect Dis. 2021;S1473-3099(20)30852-5. PMID: 34265258

17. Knoester M, Helfferich J, Poelman R, Van Leer-Buter C, Brouwer OF, Niesters HGM. Twenty-nine cases of enterovirus-D68associated acute flaccid myelitis in Europe 2016: A case series and epidemiologic overview. Pediatr Infect Dis J. 2019;38(1):1621. https://doi.org/10.1097/INF.0000000000002188 PMID: 30234793

18. Kirolos A, Mark K, Shetty J, Chinchankar N, Mcdougall C, Eunson $\mathrm{P}$, et al. Outcome of paediatric acute flaccid myelitis associated with enterovirus D68: a case series. Dev Med Child Neurol. 2019;61(3):376-80. https://doi.org/10.1111/ dmcn.14096 PMID: 30417347

19. Yea C, Bitnun A, Robinson J, Mineyko A, Barton M, Mah JK, et al. Longitudinal outcomes in the 2014 acute flaccid paralysis cluster in Canada. J Child Neurol. 2017;32(3):301-7. https://doi. org/10.1177/0883073816680770 PMID: 28193112

20. Aubart M, Gitiaux C, Roux CJ, Levy R, Schuffenecker I, Mirand A, et al. Severe acute flaccid myelitis associated with enterovirus in children: two phenotypes for two evolution profiles? Front Neurol. 2020;11:343. https://doi.org/10.3389/ fneur.2020.00343 PMID: 32411086

\section{License, supplementary material and copyright}

This is an open-access article distributed under the terms of the Creative Commons Attribution (CC BY 4.0) Licence. You may share and adapt the material, but must give appropriate credit to the source, provide a link to the licence and indicate if changes were made.

Any supplementary material referenced in the article can be found in the online version.

This article is copyright of the authors or their affiliated institutions, 2021. 